\title{
Proteome Characteristics of Liver Tissue from Patients with Parenteral Nutrition-Associated Liver Disease
}

\section{Gulsudum Mamtawla}

Department of General Surgery, Jinling Hospital, Medical School of Nanjing University

Feng Tian

Department of General Surgery, Jinling Hospital, Medical School of Nanjing University

Haifeng Sun

Department of General Surgery, Jinling Hospital, Medical School of Nanjing University

\section{Li Zhang}

Department of General Surgery, Jinlling Hospital, Medical School of Nanjing University

\section{Xuejin Gao}

Department of General Surgery, Jinling Hospital, Medical School of Nanjing University

\section{Bin Xue}

Core Laboratory, Sir Run Run Hospital, Nanjing Medical University

Xinying Wang ( $\nabla$ wangxinying@nju.edu.cn )

\section{Brief communication}

Keywords: Parenteral nutrition associated liver disease, mitochondria, oxidative phosphorylation, metabolic disorder, oxidative stress

Posted Date: January 7th, 2020

DOI: https://doi.org/10.21203/rs.2.20236/v1

License: (c) (i) This work is licensed under a Creative Commons Attribution 4.0 International License. Read Full License 


\section{Abstract}

Background Parenteral nutrition (PN)-associated liver disease (PNALD) is a common and life-threatening complication in patients receiving PN. However, its definitive etiology is not yet clear. Therefore, performed proteomic analyses of human liver tissue to explore the same.

Methods Liver tissue was derived and compared across selected patients with $(n=3)$ / without $(n=4)$ PNALD via isobaric Tag for Relative and Absolute Quantitation (iTRAQ)-based quantitative proteomics. Bioinformatics analysis was performed using Gene Ontology (GO) and Kyoto Encyclopedia of Genes and Genomes (KEGG) databases to explore the mechanisms of PNALD based on differentially expressed proteins (DEPs). The essential proteins that were differentially expressed between the two groups were explored and verified by western blotting.

Results A total of 112 proteins were found to be differentially expressed, of which 73 were downregulated, and 39 were up-regulated in the PNALD group. Bioinformatics analysis showed DEPs to be associated with mitochondrial oxidative phosphorylation (mainly involved in mitochondrial respiratory chain complex I assembly), hepatic glycolipid metabolism (involved primarily in glycogen formation and gluconeogenesis), and oxidative stress (mainly involved in antioxidant change).

Conclusion Overall, our results indicated that mitochondrial energy metabolism impairment, hepatic glycolipid metabolism disorder, and excessive oxidative stress injury might explain the comprehensive mechanism underlying PNALD. Moreover, we have provided multiple potential targets for further exploring the PNALD mechanism.

\section{Background}

Total parenteral nutrition (TPN) is a vital therapeutic measure for patients with impaired gut function, including short bowel syndrome, severe inflammatory bowel disease, or chronic idiopathic intestinal pseudo-obstruction [1-3]. Parenteral nutrition-associated liver disease (PNALD) is one of the most common complications of long-term parenteral nutrition (PN), which severely impairs the physical health of patients, and is the primary factor limiting long-term $\mathrm{PN}$ therapy.

The incidence of PNALD in infants receiving long-term parenteral nutrition is between $25-60 \%$, and that in adults is between $15 \%-40 \%$ [4]. In adults, a history of PNALD is characterized by elevated liver enzymes and associated steatosis, with ensuing complications such as steatohepatitis, cholestatic hepatitis, as well as fibrosis and cirrhosis [5]. Most biochemical abnormalities of the liver can be reversed by weaning at an early stage of PNALD, whereas most of the advanced pathophysiological changes are irreversible, resulting in cirrhosis, decompensated liver disease, liver failure, and liver carcinoma. In these patients, PNALD progresses to end-stage liver disease, requiring combined intestinal and liver transplant [6-8]. 
Research on the mechanistic pathways and ameliorative modalities in PNALD is a significant focus in the field of gastroenterology and hepatology. Although several theories have been proposed, for instance, that the components of PN directly harm the liver or that the absence of enteral nutrition disrupts the enterohepatic axis leading to liver injury, the pathophysiology and etiology of PNALD remain unclear [9], and there have been very few studies exploring the overall protein expression in liver tissue of patients with PNALD.

Proteomics is a large-scale comprehensive study of proteins, including information on protein abundance and modification, along with their interacting networks [10]. Studies regarding PNALD have been limited to the transcriptomic level in mouse models [11], and, to the best of our knowledge, neither genomic nor proteomics studies on PNALD have been conducted till date. More importantly, no prior studies have explored the use of human specimens to understand this disease. Studies are, thus, required that use liver samples from PNALD patients for proteomics research, which would be more conducive to solving clinical problems. Based on this consideration, this study aimed to explore the possible mechanisms underlying the pathogenesis of PNALD, as well as identify potential therapeutic targets by performing hepatic proteomics in patients with or without PNALD in the present study.

\section{Materials And Methods}

\section{Subjects}

Patients with diagnostic percutaneous or intraoperative liver biopsy were recruited from the Department of General Surgery, Jinling Hospital, Medical School of Nanjing University. Liver tissue was obtained from seven patients, with $(n=3)$ and without $(n=4)$ PNALD. Disease information and clinical characteristics were collected from the electronic database and detailed in Table 1. Subjects with PNALD had liver enzymes elevated 1.5 times the upper limit of normal, in the absence of any other cause, such as viral hepatitis or drug-induced changes [12]. All subjects gave their informed consent for inclusion before they participated in the study. The study was approved by the Ethics Committee of the Jinling hospital, Medical School of Nanjing University (2017NZGKJ-071).

\section{Sample preparation}

The tissues were ground in liquid nitrogen. One milliliter of lysis buffer (7M urea, 4\% SDS, 1x Protease Inhibitor Cocktail (Roche Ltd. Basel, Switzerland)) was added to samples, followed by sonication on ice and centrifugation at $13000 \mathrm{rpm}$ for $10 \mathrm{~min}$ at $4^{\circ} \mathrm{C}$. The supernatant was transferred to a fresh tube.

\section{Protein Digestion and iTRAQ Labeling}

Determine the protein concentration of the supernatant using the BCA protein assay, and then transfer $100 \mu \mathrm{g}$ protein per condition into a new tube and adjust to a final volume of $100 \mu \mathrm{L}$ with $100 \mathrm{mM}$ TEAB (triethylammonium bicarbonate). Add $5 \mu \mathrm{L}$ of the $200 \mathrm{mM}$ DTT and incubate sample at $55^{\circ} \mathrm{C}$ for 1 hour, then add $5 \mu \mathrm{L}$ of the $375 \mathrm{mM}$ iodoacetamide to the sample and incubate for 30 minutes protected from 
light at room temperature. For each sample, proteins were precipitated with ice-cold acetone, and then were redissolved in 20 $\mu \mathrm{L}$ TEAB. Then proteins were tryptically digested with sequence-grade modified trypsin (Promega, Madison, WI), and the res $\mu$ Ltant peptide mixture was labeled using chemicals from the iTRAQ reagent kit. The labeled samples were combined, desalted using C18 SPE column (Sep-Pak C18, Waters, Milford, MA) and dried in vacuo.

\section{High-pH reverse-phase separation}

The peptide mixture was re-dissolved in buffer A (buffer A: $10 \mathrm{mM}$ ammonium formate in water, $\mathrm{pH}$ 10.0, adjusted with ammonium hydroxide), and fractionated by high-pH separation using an Acquity UPLC system (Waters Corporation, Milford, MA) connected to a reverse-phase column (BEH C18 column, $2.1 \mathrm{~mm} \times 150 \mathrm{~mm}, 1.7 \mu \mathrm{m}, 300 \AA$, Waters Corporation, Milford, MA). The high-pH separation was performed using a linear gradient, starting from $0 \%$ B to $45 \%$ B in $45 \mathrm{~min}$ (B: $10 \mathrm{mM}$ ammonium formate in $90 \% \mathrm{ACN}, \mathrm{pH} 10.0$, adjusted with ammonium hydroxide). The column flow rate was maintained at 250 $\mu \mathrm{L} / \mathrm{min}$, and the column temperature was maintained at $45^{\circ} \mathrm{C}$. Twelve fractions were collected, and each was dried in a vacuum concentrator for the next step.

\section{Low-pH nano-HPLC-MS/MS analysis}

Fractions were re-suspended with $32 \mu \mathrm{l}$ solvent C (C: water with $0.1 \%$ formic acid; D: ACN with $0.1 \%$ formic acid), separated by nanoLC, and analyzed by on-line electrospray tandem mass spectrometry. Experiments were performed on a nanoACQUITY UPLC system (Waters Corporation, Milford, MA) connected to a quadrupole-Orbitrap mass spectrometer (Q-Exactive) (Thermo Fisher Scientific, Bremen, Germany) equipped with an online nano-electrospray ion source. Eight-microliter peptide sample was loaded onto the trap column (Thermo Fisher Scientific Acclaim PepMap C18, $100 \mu \mathrm{m} \times 2 \mathrm{~cm}$ ), with a flow rate of $10 \mu \mathrm{l} / \mathrm{min}$ for $3 \mathrm{~min}$, and subsequently separated on the analytical column (Acclaim PepMap C18, $75 \mu \mathrm{m} \times 25 \mathrm{~cm}$ ) with a linear gradient, from $5 \%$ D to $30 \% \mathrm{D}$ in $105 \mathrm{~min}$. The column was cleaned and reequilibrated to initial conditions for $5 \mathrm{~min}$. The column flow rate was maintained at $300 \mathrm{~nL} / \mathrm{min}$, and the column temperature was maintained at $45^{\circ} \mathrm{C}$. The electrospray voltage of $1.8 \mathrm{kV}$ versus the inlet of the mass spectrometer was used.

The Q-Exactive mass spectrometer was operated in the data-dependent mode to switch automatically between MS and MS/MS acquisition. Survey full-scan MS spectra ( $\mathrm{m} / \mathrm{z} 350-1600)$ were acquired with a mass resolution of $70 \mathrm{~K}$, followed by fifteen sequential high-energy collisional dissociations (HCD)MS/MS scans with a resolution of $17.5 \mathrm{~K}$. In all cases, one Microscan was recorded using a dynamic exclusion of $30 \mathrm{~s}$. MS/MS-fixed first mass was set at 100.

\section{Database searching and data analysis}

Tandem mass spectra were extracted by Proteome Discoverer software (Thermo Fisher Scientific, version 1.4.0.288). Charge state deconvolution and deisotoping were not performed. All MS/MS samples were analyzed using Mascot (Matrix Science, London, UK; version 2.3). Mascot was set up to search the Uniprot-SwissProt database (Taxonomy: Homo sapiens, 20245 entries) assuming the digestion enzyme 
trypsin. Mascot was searched with a fragment ion mass tolerance of $0.050 \mathrm{Da}$ and a parent ion tolerance of 10.0 PPM. Carbamidomethyl of cysteine and iTRAQ 8plex of lysine and the n-terminus were specified in Mascot as fixed modifications. Oxidation of methionine and iTRAQ 8plex of tyrosine were specified in Mascot as a variable modification. Use the percolator algorithm to control peptide level false discovery rates (FDR) lower than 1\%. Only unique peptides were used for protein quantification, and the method of normalization on protein median was used to correct experimental bias, the minimum number of proteins that must be observed to allow was set to 1000 . Bioinformatics analysis of the identified proteins was performed, and DEPs were defined in the ITRAQ experiment according to the following criteria: unique peptides $\geq 1$, P-value $<0.05$, fold change $>1.2$ or $<0.8333$ [13]. DEPs were entered into the DAVID (Database for Annotation, Visualization, and Integrated Discovery) database (david.abcc.ncifcrf.gov) for functional classification and GO enrichment analysis [14], and to determine the significant pathways according to the KEGG pathway analysis(www.kegg.jp/kegg/pathway.html). Protein-protein interaction (PPI) networks were generated through the STRING database (v10, string-db.org).

\section{Verification of proteins by western blot analysis}

Protein samples were separated by electrophoresis on $12 \%$ SDS-PAGE gel, transferred to polyvinylidene fluoride membranes, blocked with $5 \%$ non-fat milk for $1 \mathrm{~h}$, and incubated with primary antibodies against CYP2B6, DDAH1 (Thermo Scientific, Rockford, IL), FABP5 (ProteinTech, Chicago, IL, USA), CAPG, and NDUFA1 (Affinity Bioscience, USA), overnight at $4{ }^{\circ} \mathrm{C}$. Anti-rabbit horseradish peroxidase (HRP)conjugated antibody was used as a secondary antibody, followed by ECL substrate (Thermo Scientific, Rockford, IL) incubation, and image visualization using Tanon 5200 imaging system (Tanon, China). Gray-scale analysis of the bands was performed using ImageJ software. Data are presented as ratios of the target protein to the internal control.

Statistical analyses - Proteins with p-value $<0.05$ and Fold Change $<0.83$ or $>1.2$ were considered as differentially expressed proteins between the two groups. Student's t-test was used for comparison of the difference between groups.

\section{Results}

\section{Comprehensive identification of proteome in human liver tissue}

A total of 14307 peptides and 3337 proteins were identified by iTRAQ analysis. Mass spectrometry results showed 112 differentially expressed proteins (DEPs), of which 73 were down-regulated, and 39 were up-regulated in the PNALD group (Table S1).

To identify the functional classification of DEPs, this study performed gene ontology analysis with the assistance of DAVID Bioinformatics Resources, according to their molecular functions (MF), biological processes (BP), and cellular components (CC). The top 15 annotations represented in each of the three GO categories are shown in Fig. 1. Majority of enriched categories included mitochondrial and 
mitochondria-related proteins (Fig. 2-A, Table S2; total 27, 16 down-regulated and 11 up-regulated); impaired oxidative phosphorylation was the predominant process in PNALD group. Mitochondria are known to be mainly responsible for the oxidative decomposition of dextrose, fat, and protein, for providing energy. Accordingly, glycolipid metabolism (Fig. 2-B, Table S3; total 32, 28 down-regulated and 4 up-regulated) and amino acid metabolism (Fig. 2-C, Table S4; total 22, 15 down-regulated and 7 upregulated) were significantly changed between the groups.

Mitochondrial oxidative phosphorylation was impaired in PNALD patients

The mitochondrial oxidative phosphorylation system is the final biochemical pathway for the production of ATP and maintenance of cell function. Mitochondrial respiratory chain NADH dehydrogenase (complex I) is the most abundant enzyme of the electron transport chain [15], and essential for oxidative phosphorylation in mitochondria [16]. Ten subunits of NADH dehydrogenase were found to be downregulated in the PNALD group, including NDUFB11, NDUFB7, NDUFV1, NDUFA7, NDUFV2, NDUFS8, NDUFA1, NDUFS2, NDUFA12, and NDUFS1 (Table S2). Consistent with our hypothesis that mitochondrial oxidative phosphorylation in the liver of patients with PNALD might be impaired, bioinformatics analysis indicated the DEPs to be enriched in mitochondria-associated biological processes (Fig. 3), including mitochondrial electron transport (NADH to ubiquinone), response to oxidative stress, oxidation-reduction process, and ATP synthesis-coupled electron transport (Table 2).

Hepatic glycolipid metabolism disorder in patients with PNALD

The liver plays a significant role in the control of glucose homeostasis by regulating various pathways of glucose metabolism, including glycogenesis, glycogenolysis, glycolysis, and gluconeogenesis. In this study, we observed a strong enrichment of DEPs linked to metabolic enzymes. For example, phosphorylase kinases (PHKA2, PHKB, and PHKG2), which stimulate glycogen degradation [17], were down-regulated; fructose 1,6-bisphosphate 1-phosphatases (FBP1 and FBP2), the rate-limiting enzyme in gluconeogenesis [18], were down-regulated; pyruvate carboxylase (PC), which catalyzes the ATPdependent carboxylation of pyruvate to oxaloacetate, and is involved in gluconeogenesis $[19,20]$, was down-regulated. Consistent with the function of DEPs, bioinformatics analysis indicated that glycogen metabolic process (PHKA2, PHKB, PHKG2, and GNMT) and gluconeogenesis (GOT1, FBP1, FBP2, and PC) might be damaged in PNALD. Besides, we also found some glycolipid metabolism-associated DEPs, including nicotinamide N-methyltransferase (NNMT, up-regulated) [21], D-3-phosphoglycerate dehydrogenase (PHGDH, down-regulated) [22], and serine/threonine-protein phosphatase (CPPED1, down-regulated) [23].

Besides its role in glucose metabolism, the liver plays a pivotal role in lipid metabolism and is the hub of fatty acid metabolism and lipid circulation [24]. Estrogen sulfotransferase (SULT1E1), involved in the process of adipogenesis [25, 26], was found down-regulated while acetyl-CoA carboxylase 2 (ACACB), which inhibits fatty acid oxidation [27], was also down-regulated. Further, lipid metabolism-associated DEPs, including glycine N-methyltransferase (GNMT, down-regulated) [28-32], fatty acid-binding protein (FABP5, up-regulated) [33], cathepsin S (CTSS, up-regulated) [34], aldose reductase (AKR1B1, up- 
regulated) [35], and $\mathrm{N}(\mathrm{G}), \mathrm{N}(\mathrm{G})$-dimethylarginine dimethylaminohydrolase 1 (DDAH1, down-regulated) were also identified [36]. These results together indicated PNALD to be related to glycolipid metabolism disorder.

Oxidative stress caused by increased production of reactive oxygen species (ROS) may generate PNALD

Previous reports had shown oxidative stress injury to be one of the significant causes of PNALD. Production of ROS is increased when mitochondrial oxidative phosphorylation is impaired and under circumstances of antioxidant defense deficiency [37]. First, the impaired mitochondrial respiratory chain complex I assembly proteins damage the normal process of oxidative phosphorylation in patients with PNALD. Next, various antioxidant factors are down-regulated in PNALD. For example, peroxiredoxin-6 (PRDX6, down-regulated) is a peroxiredoxin that primarily functions as an antioxidant to scavenge peroxides in biological systems [38]; regucalcin (RGN, down-regulated) is an antioxidant [39]; deltaaminolevulinic acid dehydratase (ALAD, down-regulated) is an important antioxidant enzyme, whose inhibition may result in the accumulation of its substrate d-ALA, which in turn is associated with the overproduction of ROS [40]; inhibition of PHGDH (down-regulated) impairs the synthesis of heme, resulting in the impairment of oxidative phosphorylation and escape of electrons to molecular oxygen generating more ROS [41]; inhibition of aspartate aminotransferase (GOT1, downregulated) inhibits the synthesis of the NADPH antioxidant[42]; and DDAH1 (down-regulated) deficiency significantly enhances cellular oxidative stress [43]. Moreover, we found histidine metabolism-associated proteins (AMDHD1, HNMT, FTCD, HAL, and UROC1) to be down-regulated in PNALD, and previous studies had reported histidine supplementation to inhibit oxidative stress and preserve mitochondrial membrane potential as well as dehydrogenase activity [44]. Combined with the above results, we can hypothesize that oxidative stress caused by increased production of ROS may also participate in the development of PNALD.

Validation of differential expression proteins by western blotting

In order to verify the credibility of proteomics, we randomly selected several up- and down-regulated DEPS for semi-quantitative verification by western blotting, including CYP2B6, DDAH1, NDUFA1, FABP5, and CAPG (Fig. 4). As shown, expression of CYP2B6, DDAH1, and NDUFA1 was significantly down-regulated, and that of FABP 5 and CAPG was up-regulated in the PNALD group, compared to the control group. Western blotting results were in agreement with iTRAQ proteomics results.

\section{Discussion}

Total parenteral nutrition is a life-saving therapy for patients with chronic gastrointestinal failure [45]. However, related metabolic complications, especially PNALD, arise with prolonged PN [46]. This study represents the first-ever investigation of liver tissue proteomic profiles of patients with PNALD to identify potential biomarkers and elucidate the molecular alterations that occur following PNALD. We successfully identified 112 DEPs between PNALD and control groups. The number and interaction of DEPs indicated mitochondrial oxidative phosphorylation damage, glycolipid metabolism disorder, and 
oxidative stress injury to be the primary biological changes in PNALD. In addition, we performed western blot analysis and found the results to match perfectly with the iTRAQ proteomics data.

The liver is the central organ of glucose and lipid metabolism and plays a vital role in the maintenance of systemic metabolic homeostasis. When liver function is damaged, it shows abnormal regulation of glucose and lipid metabolism, including abnormal glycogen synthesis and decomposition, and gluconeogenesis, further leading to decreased blood glucose stability, thereby promoting the occurrence of PN-related glucose metabolism disorder. At the same time, lipid metabolism gets disturbed, and accumulation of lipid droplets into hepatocytes results in hepatic steatosis. Disorder of glycolipid metabolism, caused by various reasons, may predispose the individual to the development of liver steatosis, and even proceed to fibrosis, cirrhosis, and liver cancer. In our study, the patterns of glycogen metabolism and gluconeogenesis pathway in the liver of patients with PNALD were significantly altered. The enzymes responsible for gluconeogenesis in the liver of patients with PNALD, including pyruvate carboxylase and FBPase (FBP1, FBP2), were remarkably reduced. In addition, the phosphorylase kinases (PHKA2, PHKB, and PHKG) were down-regulated and stimulated glycogen degradation. Although we could not evaluate the activation of biological pathway merely by protein quantification, since the enzyme activity was not evident, we could conclude that glycogen metabolism and gluconeogenesisassociated enzymes in the liver of patients with PNALD fluctuated significantly, hence providing us with targets for the metabolic study of PNALD. Moreover, PN-related fatty liver has been one of the hotspots in recent decades; however, the underlying mechanism is still unclear. Our study may provide evidence for the mechanism of hepatic steatosis in patients with PN; for example, loss of GNMT or DDAH1 (downregulated) resulted in cholesterol accumulation in hepatocytes or higher levels of lipogenic genes, thus leading to steatosis $(33,55)$; deficiency of AKR1B1 or FABP5 (up-regulated) can repress saturated fatty acid biosynthesis and protect mice from steatosis. It can be concluded that glucose and lipid metabolism are disordered in PNALD patients, and the study on the mechanism of this disorder is limited to the thought that it is caused by intravenous glucose and lipid.

In our study, the cellular localization of DEPs (predicted using GO analysis) revealed most of the identified putative proteins to be localized in mitochondria, which may explain the disorder in glucose and lipid metabolism. Mitochondria are best known for harboring pathways involved in ATP synthesis through the tricarboxylic acid cycle and oxidative phosphorylation [47]. More than $80 \%$ of ATP is produced by mitochondrial oxidative phosphorylation. An earlier study had reported that TPN deteriorates hepatic mitochondrial function [48]. Consistent with this, our current study indicated that multiple subunits or components of the respiratory chain complex I assembly proteins, which are essential for oxidative phosphorylation in mitochondria [16], are down-regulated in PNALD. This may affect the biosynthesis of ATP, which is necessary for the maintenance of liver function. Mean ATP levels in a PN mouse model were reduced compared to those in an EN mouse, although there was no statistically significant difference between these levels [48]. Therefore, we speculated that PN might cause PNALD, at least in part, by damaging the respiratory chain complex I assembly. 
Insulin resistance is one of the metabolic phenotypes found in nonalcoholic fatty liver disease; yet, there is limited clinical evidence for insulin resistance associated with PNALD. One report in piglets had demonstrated that chronic TPN induces significant insulin resistance [29]. In agreement with the previous study, our result showed up-regulated NNMT, which correlates positively with insulin resistance [49-51], and down-regulated GNMT, deficiency of which is known to impair glucose tolerance and insulin sensitivity. Furthermore, KEGG analysis indicated the DEPs to be associated with the insulin signaling pathway and PI3K-Akt signaling pathway (Table 3). These results together suggested that insulin sensitivity was impaired in patients with PNALD. Insulin resistance was also implicated by mitochondrial dysfunction or mitochondrial oxidative stress [52]. Mitochondrial oxidative phosphorylation was hampered, proteins with antioxidant function, including PRDX6, RGN, PHGDH, ALAD, GOT1, and DDAH1, were down-regulated in patients with PNALD, and production of reactive oxygen species was increased, eventually leading to oxidative stress. Consistent with these results, several studies have demonstrated the impaired capacity of antioxidants during TPN $[53,54]$, and indicated oxidative stress to play a role in the development of PNALD [55,56].

Our results indicated that LGALS3 (Table S1), which contributes to inflammatory injury and fibrogenesis in cholestatic liver injury [57], was up-regulated, and that DEPs were associated with NOD-like receptor signaling pathway. Inflammation triggered by macrophages induced obesity-related insulin resistance [58]. Consistent with these results, previous studies had indicated that LPS-mediated macrophages and IL-1 $\beta$ production or LPS-activated Kupffer cell through TLR4 might be early events in the pathogenesis of PNALD $[59,60]$.

The present study revealed that hepatic mitochondrial oxidative phosphorylation is impaired in patients with PNALD, and may lead to glycolipid oxidative decomposition disorder, accumulation of glycolipid in the liver of patients with PNALD, thereby accelerating and aggravating hyperglycemia and fatty liver. Besides, the process of glycogen degradation, gluconeogenesis, was disordered, and hence resulted in decreased blood glucose stability, including hyperglycemia or transient hypoglycemia. Apart from mitochondrial dysfunction, high glucose or lipid levels, induced by TPN infusion, increased the production of reactive oxygen species (ROS); however, proteins with antioxidant function were down-regulated, thereby triggering oxidative stress in patients with PNALD. Besides inflammation, oxidative stress is a significant cause of insulin resistance, which, in turn, is a considerable risk factor for the development of hepatic steatosis and other metabolic diseases. Therefore, glycolipid metabolism disorder, impaired mitochondrial oxidative phosphorylation, and oxidative stress have causal relationships, eventually leading to liver dysfunction.

A limitation of this study was an insufficient number of subjects, due to the extreme rarity of PNALD and infrequent source of liver tissue. Although previous studies had reported on the abnormal glycolipid metabolism and oxidative stress in TPN, this is the first study that revealed damaged mitochondrial oxidative phosphorylation in patients with PNALD and provided a new target for exploring the mechanism and treatment of PNALD. 
In conclusion, we identified several biological abnormalities that occur in patients with PNALD, such as in glycolipid metabolism, mitochondrial oxidative phosphorylation, and oxidative stress. We also detected the involvement of insulin and inflammatory signaling in PNALD pathogenesis. Our results provided new insights into the changes that occur in PNALD, and further identified candidate proteins as future PNALD biomarkers or therapeutic targets.

\section{Abbreviations}

Parenteral nutrition, PN; Parenteral nutrition-associated liver disease, PNALD; Isobaric Tag for Relative and Absolute Quantitation, iTRAQ; Gene Ontology, GO; Kyoto Encyclopedia of Genes and Genomes, KEGG; Differentially expressed proteins, DEPs; Total parenteral nutrition, TPN; Molecular functions, MF; Biological processes, BP; Cellular components, CC; Acetyl-CoA carboxylase 2, ACACB; Aldose reductase, AKR1B1; Delta-aminolevulinic acid dehydratase, ALAD; Probable imidazolonepropionase, AMDHD1; Macrophage-capping protein, CAPG; Serine/threonine-protein phosphatase, CPPED1; Cathepsin S, CTSS; Cytochrome P450 2B6, CYP2B6; N(G), N(G)-dimethylarginine; Dimethylaminohydrolase 1, DDAH1; Fatty acid-binding protein, FABP5; Fructose-1,6-bisphosphatase 1, FBP1; Fructose-1,6-bisphosphatase isozyme 2, FBP2; Formimidoyltransferase-cyclodeaminase, FTCD; Glycine N-methyltransferase; GNMT; Aspartate aminotransferase, GOT1; Aspartate aminotransferase, cytoplasmic, GOT1; Histidine ammonia-lyase, HAL; Histamine N-methyltransferase, HNMT; Interleukin-1 $\beta$, IL-1 $\beta$; Relative and Absolute Quantitation, iTRAQ; Galectin-3, LGALS3; Lipopolysaccharide, LPS; Molecular function, MF; NADH dehydrogenase [ubiquinone] 1 alpha subcomplex subunit 1, NDUFA1; NADH dehydrogenase [ubiquinone] 1 alpha subcomplex subunit 12, NDUFA12; NADH dehydrogenase [ubiquinone] 1 alpha subcomplex subunit 7, NDUFA7; NADH dehydrogenase [ubiquinone] 1 beta subcomplex subunit 11, NDUFB11; NADH dehydrogenase [ubiquinone] 1 beta subcomplex subunit 7, NDUFB7; NADH-ubiquinone oxidoreductase $75 \mathrm{kDa}$ subunit, mitochondrial, NDUFS1; NADH dehydrogenase [ubiquinone] iron-sulfur protein 2, NDUFS2; NADH dehydrogenase [ubiquinone] iron-sulfur protein 8, NDUFS8; NADH dehydrogenase [ubiquinone] flavoprotein 1, NDUFV1; NADH dehydrogenase [ubiquinone] flavoprotein 2, mitochondrial, NDUFV2; Nicotinamide N-methyltransferase, NNMT; Pyruvate carboxylase, PC; D-3-phosphoglycerate dehydrogenase, PHGDH; Phosphorylase b kinase regulatory subunit alpha, liver isoform, PHKA2; Phosphorylase b kinase regulatory subunit beta, PHKB; Phosphorylase b kinase gamma catalytic chain, liver/testis isoform, PHKG2; Peroxiredoxin-6, PRDX6; Regucalcin, RGN; Reactive oxygen species, ROS; Estrogen sulfotransferase, SULT1E1; Toll-like receptor 4, TLR4; Total parenteral nutrition, TPN; Urocanate hydratase, UROC1.

\section{Declarations}

Ethics approval and consent to participate $₫ A \| l l$ subjects gave their informed consent for inclusion before they participated in the study. The study was approved by the Ethics Committee of the Jinling hospital, Medical School of Nanjing University (2017NZGKJ-071).

Consent for publicationロNot applicable. 
Availability of data and materials: All data generated or analyzed during this study are included in this published article (and its supplementary information files).

Competing interests: The authors declare that they have no competing interests.

Funding: This work gained support from the National Natural Science Foundation of China (General Program, 81770531), the Excellent Youth Foundation Project of Jiangsu Province (BK20170009), the Key Medical Talents Foundation of Jiangsu Province (ZDRCA2016091), the National Natural Science Foundation of China $(81700518,81900524)$, and the Natural Science Foundation of Jiangsu Province (BK20170622).

\section{Author Contributions:}

W.X.Y. and T.F. designed the study. T.F., S.H.F., Z.L., and G.X.J. collected the samples. M.G. performed the experiments. M.G. and S.H.F. analyzed the data and wrote the manuscript. W.X.Y. and X.B.

reviewed/edited the manuscript. W.X.Y. and X.B. supervised the research. All authors read and approved the final manuscript.

Acknowledgements: We express our gratitude to all of the participants who consented to participate in this study.

Supplementary information: Supplementary tables and other bioinformation analysis (Annotation Enrichment Analysis, Different Proteins Analysis, Hierarchical Clustering Analysis, KEGG Analysis and Network Analysis) were listed in the file.

\section{References}

1. Stanghellini V CRDG: Natural history of intestinal failure induced by chronic idiopathic intestinal pseudo-obstruction. Transplantation proceedings 2010;42:15-18.

2. Triantafillidis JK, Papalois AE: The role of total parenteral nutrition in inflammatory bowel disease: current aspects. Scand J Gastroenterol 2014;49:3-14.

3. Ekema G, Milianti S, Boroni G: Total parenteral nutrition in patients with short bowel syndrome. Minerva Pediatrica 2009;61:283-291.

4. Beath SV, Kelly DA: Total Parenteral Nutrition-Induced Cholestasis: Prevention and Management. Clinics in Liver Disease 2016;20:159-176.

5. G O, C M, C V, N C, A G, P V: Pediatric parenteral nutrition-associated liver disease and cholestasis: Novel advances in pathomechanisms-based prevention and treatment. Digestive and liver disease : 
official journal of the Italian Society of Gastroenterology and the Italian Association for the Study of the Liver 2016;48:215-222.

6. D S, D B, M R, V L, M B, K S, L D, M S: Improved outcomes in paediatric intestinal failure with aggressive prevention of liver disease. European journal of pediatric surgery : official journal of Austrian Association of Pediatric Surgery ... [et al] = Zeitschrift fur Kinderchirurgie 2009;19:348-353.

7. Koseesirikul P, Chotinaruemol S, Ukarapol N: Incidence and risk factors of parenteral nutritionassociated liver disease in newborn infants. Pediatrics International 2012;54:434-436.

8. L A, DT D, MA B, GL F, M P, KM G: Intravenous Fat Emulsion Formulations for the Adult and Pediatric Patient: Understanding the Differences. Nutrition in clinical practice : official publication of the American Society for Parenteral and Enteral Nutrition 2016;31:596-609.

9. C D, A P, J F, C M, K B, M W, V K, W P, J K, S AM, H O, AK J: Role of the GutखLiver Axis in Driving Parenteral Nutrition-Associated Injury. Children (Basel, Switzerland) 2018;5:undefined.

10. NL A, NG A: Proteome and proteomics: new technologies, new concepts, and new words. Electrophoresis 1998;19:1853-1861.

11. L Z, I Y, B K, J S, L G, N M, BT B, GL G: Dysregulation of bile acid homeostasis in parenteral nutrition mouse model. American journal of physiology. Gastrointestinal and liver physiology 2016;310:G93G102.

12. Beath SV, Kelly DA: Total Parenteral Nutrition-Induced Cholestasis. Clinics in Liver Disease 2016;20:159-176.

13. UniProt: a hub for protein information. Nucleic acids research 2015;43:D204-D212.

14. G D, BT S, DA H, J Y, W G, HC L, RA L: DAVID: Database for Annotation, Visualization, and Integrated Discovery. Genome biology 2003;4:P3.

15. Chaban Y, Boekema EJ, Dudkina NV: Structures of mitochondrial oxidative phosphorylation supercomplexes and mechanisms for their stabilisation. Biochimica et Biophysica Acta (BBA) Bioenergetics 2014;1837:418-426.

16. Formosa LE, Dibley MG, Stroud DA, Ryan MT: Building a complex complex: assembly of mitochondrial respiratory chain complex I. Seminars in Cell \& Developmental Biology 2017:S428657638.

17. Skamnaki VT, Owen DJ, Noble MEM, Lowe ED, Lowe G, Oikonomakos NG, Johnson LN: Catalytic Mechanism of Phosphorylase Kinase Probed by Mutational Studiest,ł. Biochemistry 1999;38:1471814730.

18. Kaur R, Dahiya L, Kumar M: Fructose-1,6-bisphosphatase inhibitors: A new valid approach for management of type 2 diabetes mellitus. European Journal of Medicinal Chemistry 2017;141:473505.

19. Jitrapakdee S, St Maurice M, Rayment I, Cleland WW, Wallace JC, Attwood PV: Structure, mechanism and regulation of pyruvate carboxylase. Biochemical Journal 2008;413:369-387. 
20. Targeting Pyruvate Carboxylase Reduces Gluconeogenesis and Adiposity and Improves Insulin Resistance.

21. Pissios P: Nicotinamide N -Methyltransferase: More Than a Vitamin B3 Clearance Enzyme. Trends in Endocrinology \& Metabolism 2017;28:340-353.

22. Okabe K, Usui I, Yaku K, Hirabayashi Y, Tobe K, Nakagawa T: Deletion of PHGDH in adipocytes improves glucose intolerance in diet-induced obese mice. Biochemical and Biophysical Research Communications 2018;504:309-314.

23. Vaittinen M, Kaminska D, Kakela P, Eskelinen M, Kolehmainen M, Pihlajamaki J, Uusitupa M, Pulkkinen L: Downregulation of CPPED1 Expression Improves Glucose Metabolism In Vitro in Adipocytes. Diabetes 2013;62:3747-3750.

24. Nguyen P, Leray V, Diez M, Serisier S, Bloc H JL, Siliart B, Dumon H: Liver lipid metabolism. Journal of Animal Physiology and Animal Nutrition 2008;92:272-283.

25. Wada T, Ihunnah CA, Gao J, Chai X, Zeng S, Philips BJ, Rubin JP, Marra KG, Xie W: Estrogen Sulfotransferase Inhibits Adipocyte Differentiation. Molecular Endocrinology 2011;25:1612-1623.

26. Ihunnah CA, Wada T, Philips BJ, Ravuri SK, Gibbs RB, Kirisci L, Rubin JP, Marra KG, Xie W: Estrogen Sulfotransferase/SULT1E1 Promotes Human Adipogenesis. Molecular and Cellular Biology 2014;34:1682-1694.

27. Murea M, Freedman BI, Parks JS, Antinozzi PA, Elbein SC, Ma L: Lipotoxicity in Diabetic Nephropathy: The Potential Role of Fatty Acid Oxidation. Clinical Journal of the American Society of Nephrology 2010;5:2373-2379.

28. Chen YM, Shiu JY, Tzeng SJ, Shih LS, Chen YJ, Lui WY, Chen PH: Characterization of glycine-Nmethyltransferase-gene expression in human hepatocellular carcinoma. Int J Cancer 1998;75:787793.

29. Stoll B, Horst DA, Cui L, Chang X, Ellis KJ, Hadsell DL, Suryawan A, Kurundkar A, Maheshwari A, Davis TA, Burrin DG: Chronic Parenteral Nutrition Induces Hepatic Inflammation, Steatosis, and Insulin Resistance in Neonatal Pigs. The Journal of Nutrition 2010;140:2193-2200.

30. Liao YJ, Chen TL, Lee TS, Wang HA, Wang CK, Liao LY, Liu RS, Huang SF, Chen YM: Glycine Nmethyltransferase deficiency affects Niemann-Pick type $\mathrm{C} 2$ protein stability and regulates hepatic cholesterol homeostasis. Mol Med 2012;18:412-422.

31. Liu S, Li Y, Chen Y, Chiang E, Li AF, Lee Y, Tsai T, Hsiao M, Hwang S, Chen YA: Glycine Nmethyltransferase-/- mice develop chronic hepatitis and glycogen storage disease in the liver. Hepatology 2007;46:1413-1425.

32. Martínez-Chantar ML, Vázquez-Chantada M, Ariz U, Martínez N, Varela M, Luka Z, Capdevila A, Rodríguez J, Aransay AM, Matthiesen R, Yang H, Calvisi DF, Esteller M, Fraga M, Lu SC, Wagner C, Mato JM: Loss of the glycine N-methyltransferase gene leads to steatosis and hepatocellular carcinoma in mice. Hepatology 2008;47:1191-1199.

33. Hotamisligil GS, Bernlohr DA: Metabolic functions of FABPs--mechanisms and therapeutic implications. Nat Rev Endocrinol 2015;11:592-605. 
34. Multiple Statistical Methods for Assessing Diff erential Gene Expression in Microarray Data of Diabetic Model Rats to Predict the Molecular Mechanism of Atorvastatin on Anti-Atherogenesis.

35. KE B, KA B, MM M, RD K, CJ F, SK S, EM B: Immunodetection of aldose reductase in normal and diseased human liver. Histology and histopathology 2005;20:429-436.

36. Li T, Feng R, Zhao C, Wang Y, Wang J, Liu S, Cao J, Wang H, Wang T, Guo Y, Lu Z: Dimethylarginine Dimethylaminohydrolase 1 Protects Against High-Fat Diet-Induced Hepatic Steatosis and Insulin Resistance in Mice. Antioxidants \& Redox Signaling 2017;26:598-609.

37. Chow J, Rahman J, Achermann JC, Dattani MT, Rahman S: Mitochondrial disease and endocrine dysfunction. Nature Reviews Endocrinology 2017;13:92-104.

38. Patel P, Chatterjee S: Peroxiredoxin6 in Endothelial Signaling. Antioxidants 2019;8:63.

39. Yamaguchi M, Murata T: Involvement of regucalcin in lipid metabolism and diabetes. Metabolism 2013;62:1045-1051.

40. B P, R C, E K, EJ B: 5-aminolevulinic acid-induced alterations of oxidative metabolism in sedentary and exercise-trained rats. Journal of applied physiology (Bethesda, Md. : 1985) 1992;72:226-230.

41. Vandekeere S, Dubois C, Kalucka J, Sullivan MR, García-Caballero M, Goveia J, Chen R, Diehl FF, BarLev L, Souffreau J, Pircher A, Kumar S, Vinckier S, Hirabayashi Y, Furuya S, Schoonjans L, Eelen G, Ghesquière B, Keshet E, Li X: Serine Synthesis via PHGDH Is Essential for Heme Production in Endothelial Cells. Cell Metabolism 2018;28:573-587.

42. Hong C, Zheng J, Li X: Inhibition of GOT1 sensitizes colorectal cancer cells to 5-fluorouracil. Cancer Chemotherapy and Pharmacology 2017;79:835-840.

43. Zhao C, Li T, Han B, Yue W, Shi L, Wang H, Guo Y, Lu Z: DDAH1 deficiency promotes intracellular oxidative stress and cell apoptosis via a miR-21-dependent pathway in mouse embryonic fibroblasts. Free Radical Biology and Medicine 2016;92:50-60.

44. Ommati MM, Jamshidzadeh A, Heidari R, Sun Z, Zamiri MJ, Khodaei F, Mousapour S, Ahmadi F, Javanmard N, Shirazi Yeganeh B: Carnosine and Histidine Supplementation Blunt Lead-Induced Reproductive Toxicity through Antioxidative and Mitochondria-Dependent Mechanisms. Biological Trace Element Research 2019;187:151-162.

45. Dudrick SJ, Palesty JA: Historical Highlights of the Development of Total Parenteral Nutrition. Surg Clin North Am 2011;91:693-717.

46. Mutanen A, Lohi J, Heikkilä P, Jalanko H, Pakarinen MP: Liver Inflammation Relates to Decreased Canalicular Bile Transporter Expression in Pediatric Onset Intestinal Failure. Annals of Surgery 2017;268:1.

47. van der Bliek AM, Sedensky MM, Morgan PG: Cell Biology of the Mitochondrion. Genetics 2017;207:843-871.

48. Katayama T, Tanaka M, Tanaka K, Asonuma K, Uemoto S, Okamura R, Utsunomiya H, Fujita S, Ueda J, Tanaka A, Ozawa K: Alterations in Hepatic Mitochondrial Function during Total Parenteral Nutrition in Immature Rats. Journal of Parenteral and Enteral Nutrition 1990;14:640-645. 
49. Riederer M, Erwa W, Zimmermann R, Frank S, Zechner R: Adipose tissue as a source of nicotinamide N-methyltransferase and homocysteine. Atherosclerosis 2009;204:412-417.

50. Kannt A, Pfenninger A, Teichert L, Tönjes A, Dietrich A, Schön MR, Klöting N, Blüher M: Association of nicotinamide-N-methyltransferase mRNA expression in human adipose tissue and the plasma concentration of its product, 1-methylnicotinamide, with insulin resistance. Diabetologia 2015;58:799-808.

51. Kraus D, Yang Q, Kong D, Banks AS, Zhang L, Rodgers JT, Pirinen E, Pulinilkunnil TC, Gong F, Wang Y, Cen Y, Sauve AA, Asara JM, Peroni OD, Monia BP, Bhanot S, Alhonen L, Puigserver P, Kahn BB: Nicotinamide N-methyltransferase knockdown protects against diet-induced obesity. Nature 2014;508:258-262.

52. Fazakerley DJ, Minard AY, Krycer JR, Thomas KC, Stöckli J, Harney DJ, Burchfield JG, Maghzal GJ, Caldwell ST, Hartley RC, Stocker R, Murphy MP, James DE: Mitochondrial oxidative stress causes insulin resistance without disrupting oxidative phosphorylation. Journal of Biological Chemistry 2018;293:7315-7328.

53. Matilla B, Ortíz J, González P, García-Díez F, Jorquera F, Culebras JM, González-Gallego J, Tuñón MJ: Effects of parenteral nutrition supplemented with glutamine or glutamine dipeptides on liver antioxidant and detoxication systems in rats. Nutrition 2000;16:125-128.

54. RJ S, SF T, MW D, R K, NJ S, RH S, G M, Alimentation HDOC, However TUMC, Rats. TWFB: Hepatic oxidant injury and glutathione depletion during total parenteral nutrition in weanling rats. The American journal of physiology 1996;270:G691-G700.

55. Staun M, Pironi L, Bozzetti F, Baxter J, Forbes A, Joly F, Jeppesen P, Moreno J, Hébuterne X, Pertkiewicz M, Mühlebach S, Shenkin A, Van Gossum A: ESPEN Guidelines on Parenteral Nutrition: Home Parenteral Nutrition (HPN) in adult patients. Clinical Nutrition 2009;28:467-479.

56. Cai W, Wu J, Hong L, Xu Y, Tang Q, Shi C: Oxidative injury and hepatocyte apoptosis in total parenteral nutrition-associated liver dysfunction. Journal of Pediatric Surgery 2006;41:1663-1668.

57. Tian J, Yang G, Chen H, Hsu DK, Tomilov A, Olson KA, Dehnad A, Fish SR, Cortopassi G, Zhao B, Liu F, Gershwin ME, Török NJ, Jiang JX: Galectin-3 regulates inflammasome activation in cholestatic liver injury. The FASEB Journal 2016;30:4202-4213.

58. Lauterbach MAR, Wunderlich FT: Macrophage function in obesity-induced inflammation and insulin resistance. Pflügers Archiv - European Journal of Physiology 2017;469:385-396.

59. El Kasmi KC, Vue PM, Anderson AL, Devereaux MW, Ghosh S, Balasubramaniyan N, Fillon SA, Dahrenmoeller C, Allawzi A, Woods C, McKenna S, Wright CJ, Johnson L, D Alessandro A, Reisz JA, Nozik-Grayck E, Suchy FJ, Sokol RJ: Macrophage-derived IL-1ß/NF-kB signaling mediates parenteral nutrition-associated cholestasis. Nature Communications 2018;9.

60. El Kasmi KC, Anderson AL, Devereaux MW, Fillon SA, Harris JK, Lovell MA, Finegold MJ, Sokol RJ: Toll-like receptor 4-dependent Kupffer cell activation and liver injury in a novel mouse model of parenteral nutrition and intestinal injury. Hepatology 2012;55:1518-1528. 


\section{Tables}

Table1. Demographic and Clinical Characteristics of Participants

\begin{tabular}{|c|c|c|c|c|c|c|c|c|}
\hline Group & & PNALD & & & & n-PNALD & & \\
\hline Characteristic & Pt. 1 & Pt. 2 & Pt. 3 & Pt. (A) & Pt. (B) & Pt. (C) & Pt. (D) & $\begin{array}{l}\mathrm{P} \text { - } \\
\text { value }\end{array}$ \\
\hline Age (yr.) & 58 & 49 & 31 & 46 & 52 & 62 & 38 & 0.5545 \\
\hline Sex & man & man & man & man & man & Woman & Woman & - \\
\hline Weight (kg) & 60 & 61 & 43 & 72 & 76 & 60 & 52 & 0.2604 \\
\hline $\mathrm{BMI}, \mathrm{kg} / \mathrm{m} 2$ & 21.6 & 20.6 & 15.8 & 23.2 & 24.5 & 23.1 & 20.1 & 0.1277 \\
\hline Diagnosis & $\begin{array}{l}\text { short bowel } \\
\text { syndrome }\end{array}$ & $\begin{array}{l}\text { short bowel } \\
\text { syndrome }\end{array}$ & $\begin{array}{l}\text { abdominal } \\
\text { cocoon }\end{array}$ & $\begin{array}{c}\text { hepatic } \\
\text { hemangioma }\end{array}$ & Cholelithiasis & $\begin{array}{c}\text { hepatic } \\
\text { hemangioma }\end{array}$ & $\begin{array}{c}\text { hepatic } \\
\text { hemangioma }\end{array}$ & - \\
\hline ALT (U/L) & 136 & 129 & 217 & 22 & 32 & 34 & 31 & 0.0027 \\
\hline AST (U/L) & 132 & 125 & 257 & 26 & 28 & 21 & 29 & 0.0099 \\
\hline GGT (U/L) & 335 & 281 & 458 & 39 & 41 & 36 & 27 & 0.0007 \\
\hline TBIL (umol/L) & 60.7 & 45.4 & 45.5 & 6.9 & 10.2 & 8.3 & 7.7 & 0.0003 \\
\hline $\mathrm{BUN}(\mathrm{mmol} / \mathrm{L})$ & 8.9 & 14.1 & 9.5 & 3.4 & 4.6 & 3.9 & 5.2 & 0.0063 \\
\hline Scr (umol/L) & 50 & 189.8 & 68 & 79 & 83 & 69 & 77 & 0.5187 \\
\hline Days with PN & 123 & 109 & 138 & 0 & 0 & 0 & 0 & - \\
\hline
\end{tabular}

ALT, aspartate aminotransferase; AST, alanine aminotransferase; GGT, gamma-glutamyl transferase; TBIL, total bilirubin; BUN, blood urea nitrogen; Scr, serum creatinine; Pt, patient. 


\begin{tabular}{|c|c|c|c|c|}
\hline \multirow[t]{2}{*}{ Analysis } & \multirow[t]{2}{*}{ Pathway } & \multicolumn{3}{|l|}{ Protein number } \\
\hline & & List & Up & Down \\
\hline \multirow{7}{*}{$\begin{array}{l}\text { Cellular } \\
\text { Component }\end{array}$} & mitochondrion & NDUFB11, GRPEL1, DNM1L, NDUFB7, PSAP, NDUFA7, ACACB, VDAC2, & 8 & 13 \\
\hline & & $\begin{array}{l}\text { NDUFA1, GLYCTK, NMT1, YWHAH, GOT1, NDUFV2, NDUFS8, SLC25A3, } \\
\text { BDH2, DDAH1, NDUFS2, NDUFS1, PC }\end{array}$ & & \\
\hline & $\begin{array}{l}\text { mitochondrial respiratory chain } \\
\text { complex I }\end{array}$ & $\begin{array}{c}\text { NDUFB11, NDUFB7, NDUFV1, NDUFA7, NDUFV2, NDUFS8, NDUFA1, } \\
\text { NDUFS2, NDUFA12, NDUFS1 }\end{array}$ & 0 & 10 \\
\hline & mitochondrial inner membrane & $\begin{array}{l}\text { NDUFB11, LGALS3, NDUFB7, NDUFV1, NDUFA7, NDUFV2, SLC25A3, } \\
\text { VDAC2, NDUFA1, NDUFA12 }\end{array}$ & 3 & 7 \\
\hline & mitochondrial matrix & GRPEL1, NDUFS8, PDHX, AGXT, NDUFS2, NDUFS1, PC & 4 & 3 \\
\hline & mitochondrial outer membrane & DNM1L, ACACB, VDAC2 & 2 & 1 \\
\hline & mitochondrial intermembrane space & NDUFB7, NDUFS1 & 0 & 2 \\
\hline \multirow[t]{7}{*}{$\begin{array}{l}\text { Molecular } \\
\text { function }\end{array}$} & $\begin{array}{l}\text { NADH dehydrogenase (ubiquinone) } \\
\text { activity }\end{array}$ & $\begin{array}{l}\text { NDUFB7, NDUFV1, NDUFA7, NDUFV2, NDUFS8, NDUFA1, NDUFS2, } \\
\text { NDUFA12, NDUFS1 }\end{array}$ & 0 & 9 \\
\hline & electron carrier activity & $\begin{array}{l}\text { NDUFV2, AKR1B1, PHGDH, HAAO, SH3BGRL3, NDUFS2, NDUFA12, } \\
\text { NDUFS1 }\end{array}$ & 2 & 6 \\
\hline & NAD binding & AHCY, SORD, NDUFV1, PHGDH, BDH2, NDUFS2 & 0 & 6 \\
\hline & NADH dehydrogenase activity & NDUFV1, NDUFS8, NDUFS2 & 0 & 3 \\
\hline & $\begin{array}{l}\text { oxidoreductase activity, acting on } \\
\qquad \mathrm{NAD}(\mathrm{P}) \mathrm{H}\end{array}$ & NDUFS8, NDUFS2, NDUFS1 & 0 & 3 \\
\hline & oxidoreductase activity & PTGR2, SORD, NDUFV2, AKR1B1, ADH1C, BDH2 & 0 & 6 \\
\hline & $\begin{array}{l}\text { oxidoreductase activity, acting on } \\
\text { the CH-CH group of donors, NAD or } \\
\text { NADP as acceptor }\end{array}$ & SRD5A3, BDH2 & 0 & 2 \\
\hline \multirow[t]{6}{*}{$\begin{array}{l}\text { Biological } \\
\text { process }\end{array}$} & $\begin{array}{l}\text { mitochondrial electron transport, } \\
\text { NADH to ubiquinone }\end{array}$ & $\begin{array}{l}\text { NDUFB11, NDUFB7, NDUFV1, NDUFA7, NDUFV2, NDUFS8, NDUFA1, } \\
\text { NDUFS2, NDUFA12, NDUFS1 }\end{array}$ & 0 & 10 \\
\hline & $\begin{array}{l}\text { mitochondrial respiratory chain } \\
\text { complex I assembly }\end{array}$ & $\begin{array}{l}\text { NDUFB11, NDUFB7, NDUFV1, NDUFA7, NDUFV2, NDUFS8, NDUFA1, } \\
\text { NDUFS2, NDUFA12, NDUFS1 }\end{array}$ & 0 & 10 \\
\hline & response to oxidative stress & EGFR, ALAD, ATOX1, NDUFS8, NDUFS2, NDUFA12, NAPRT & 1 & 6 \\
\hline & oxidation-reduction process & $\begin{array}{l}\text { PTGR2, SORD, CYP2B6, PCBD1, HGD, CRYL1, CBR1, PRDX6, AKR1B1, } \\
\text { FMO3, SRD5A3, HAAO, PHGDH, SH3BGRL3 }\end{array}$ & 3 & 11 \\
\hline & steroid metabolic process & CYP2B6, SULT1B1, SULT1A1, SULT1E1 & 0 & 4 \\
\hline & $\begin{array}{l}\text { ATP synthesis coupled electron } \\
\text { transport }\end{array}$ & NDUFA7, NDUFS1 & 0 & 2 \\
\hline
\end{tabular}


Table 3. KEGG pathways of differentially expressed proteins

\begin{tabular}{|c|c|c|c|c|c|c|}
\hline \multirow{2}{*}{$\begin{array}{c}\text { S. } \\
\text { No. }\end{array}$} & & \multirow[t]{2}{*}{ KEGG ID } & \multirow[t]{2}{*}{ Pathway } & \multicolumn{3}{|c|}{ Protein number } \\
\hline & & & & List & Up & Down \\
\hline 1 & Energy metabolism & 00190 & Oxidative phosphorylation & 11 & 1 & 10 \\
\hline \multirow[t]{9}{*}{2} & Carbohydrate metabolism & 01200 & Carbon metabolism & 8 & 2 & 6 \\
\hline & & 00030 & Pentose phosphate pathway & 4 & 0 & 4 \\
\hline & & 00040 & Pentose and glucuronate interconversions & 4 & 1 & 3 \\
\hline & & 00051 & Fructose and mannose metabolism & 4 & 1 & 3 \\
\hline & & 00010 & Glycolysis / Gluconeogenesis & 3 & 0 & 3 \\
\hline & & 00052 & Galactose metabolism & 2 & 1 & 1 \\
\hline & & 00520 & Amino sugar and nucleotide sugar metabolism & 2 & 1 & 1 \\
\hline & & 00620 & Pyruvate metabolism & 2 & 1 & 1 \\
\hline & & 00630 & Glyoxylate and dicarboxylate metabolism & 2 & 1 & 1 \\
\hline \multirow[t]{7}{*}{3} & Amino acid metabolism & 01230 & Biosynthesis of amino acids & 5 & 1 & 4 \\
\hline & & 00260 & Glycine, serin vitamin e and threonine metabolism & 5 & 1 & 4 \\
\hline & & 00340 & Histidine metabolism & 5 & 0 & 5 \\
\hline & & 00350 & Tyrosine metabolism & 3 & 0 & 3 \\
\hline & & 00250 & Alanine, aspartate and glutamate metabolism & 3 & 1 & 2 \\
\hline & & 00270 & Cysteine and methionine metabolism & 3 & 0 & 3 \\
\hline & & 00220 & Arginine biosynthesis & 2 & 0 & 2 \\
\hline \multirow[t]{3}{*}{4} & Lipid metabolism & 00590 & Arachidonic acid metabolism & 2 & 0 & 2 \\
\hline & & 00561 & Glycerolipid metabolism & 2 & 1 & 1 \\
\hline & & 00140 & Steroid hormone biosynthesis & 2 & 0 & 2 \\
\hline \multirow[t]{3}{*}{5} & Metabolism of cofactors and vitamins & 00790 & Folate biosynthesis & 4 & 1 & 3 \\
\hline & & 00760 & Nicotinate and nicotinamide metabolism & 2 & 1 & 1 \\
\hline & & 00830 & Retinol metabolism & 2 & 0 & 2 \\
\hline \multirow[t]{3}{*}{6} & Xenobiotics biodegradation and metabolism & 00982 & Drug metabolism - cytochrome P450 & 3 & 1 & 2 \\
\hline & & 00980 & Metabolism of xenobiotics by cytochrome P 450 & 3 & 0 & 3 \\
\hline & & 00983 & Drug metabolism - other enzymes & 2 & 0 & 2 \\
\hline 7 & Nucleotide metabolism & 00240 & Pyrimidine metabolism & 2 & 0 & 2 \\
\hline \multirow[t]{3}{*}{8} & Signal transduction & 04020 & Calcium signaling pathway & 5 & 1 & 4 \\
\hline & & 04152 & AMPK signaling pathway & 3 & 0 & 2 \\
\hline & & 04151 & PI3K-Akt signaling pathway & 2 & 1 & 1 \\
\hline 9 & Nervous system & 04723 & Retrograde endocannabinoid signaling & 10 & 0 & 10 \\
\hline \multirow[t]{3}{*}{10} & Neurodegenerative diseases & 05012 & Parkinson's disease & 11 & 1 & 10 \\
\hline & & 05016 & Huntington's disease & 11 & 1 & 10 \\
\hline & & 05010 & Alzheimer's disease & 10 & 0 & 10 \\
\hline \multirow[t]{2}{*}{11} & Endocrine system & 04910 & Insulin signaling pathway & 6 & 0 & 6 \\
\hline & & 04922 & Glucagon signaling pathway & 5 & 0 & 5 \\
\hline \multirow[t]{3}{*}{12} & Endocrine and metabolic diseases & 04932 & Non-alcoholic fatty liver disease (NAFLD) & 10 & 0 & 10 \\
\hline & S. & KEGG ID & Pathway & Pro & ein nu & mber \\
\hline & No. & & & List & Up & Down \\
\hline \multirow[t]{3}{*}{13} & Transport and catabolism & 04142 & Lysosome & 5 & 5 & 0 \\
\hline & & 04144 & Endocytosis & 2 & 0 & 2 \\
\hline & & 04145 & Phagosome & 2 & 2 & 0 \\
\hline \multirow[t]{3}{*}{14} & Cancers & 05204 & Chemical carcinogenesis & 3 & 0 & 3 \\
\hline & & 05205 & Proteoglycans in cancer & 2 & 1 & 1 \\
\hline & & 05200 & Pathways in cancer & 2 & 0 & 2 \\
\hline \multirow[t]{3}{*}{15} & Cell growth and death & 04114 & Oocyte meiosis & 2 & 1 & 1 \\
\hline & & 04110 & Cell cycle & 2 & 1 & 1 \\
\hline & & 04217 & Necroptosis & 2 & 2 & 0 \\
\hline 16 & Cell motility & 04810 & Regulation of actin cytoskeleton & 2 & 1 & 1 \\
\hline 17 & Immune system & 04612 & Antigen processing and presentation & 2 & 2 & 0 \\
\hline
\end{tabular}

Page $18 / 22$ 


\begin{tabular}{|c|c|c|c|c|c|c|}
\hline & & $\begin{array}{l}04621 \\
04610\end{array}$ & $\begin{array}{l}\text { NOD-like receptor signaling pathway } \\
\text { Complement and coagulation cascades }\end{array}$ & $\begin{array}{l}2 \\
2\end{array}$ & $\begin{array}{l}2 \\
2\end{array}$ & $\begin{array}{l}0 \\
0\end{array}$ \\
\hline 18 & Infectious diseases & 05120 & Epithelial cell signaling in Helicobacter pylori infection & 2 & 1 & 1 \\
\hline 19 & Digestive system & 04976 & Bile secretion & 2 & 0 & 2 \\
\hline 20 & Excretory system & 04966 & Collecting duct acid secretion & 2 & 1 & 1 \\
\hline
\end{tabular}

\section{Figures}

\section{GO-Analysis-BP - Log10(P-Value)}

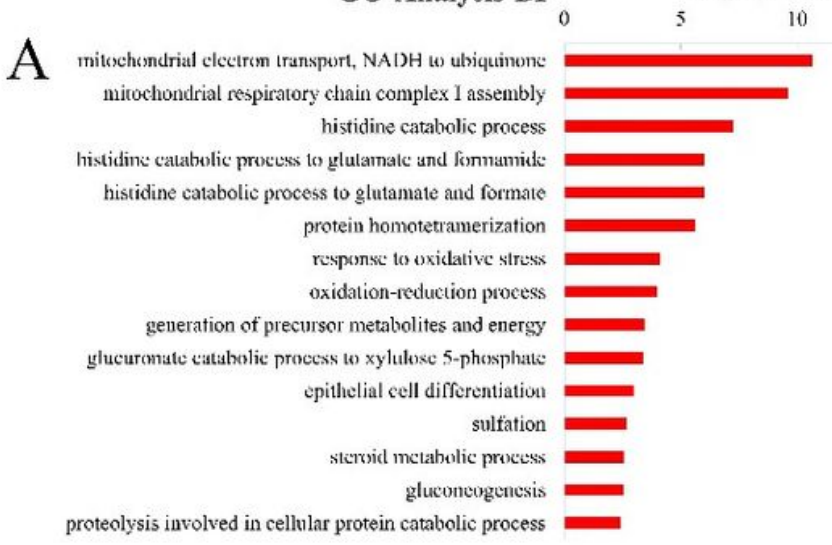
proteolysis involved in cellular protein catabolic process

\section{GO-Analysis-CC}

B

$$
\text { cytosol }
$$

0 myelin sheath

mitochondrion

cytoplasm

phosphorylase kinase complex mitochondrial inner membrane

lysosomal lumen

melanosome microvillus mitochondrial matrix extracellular space blood microparticle

\section{GO-Analysis-MF $\quad-\log 10(P$-value $)$}

$\begin{array}{llcccc}0 & 2 & 4 & 6 & 8 & 10\end{array}$

C VAIII dehydrogenase (ubiquinone) activity electron carier activity $\mathrm{NAD}$ binding identical protein binding phosphorylasc kinase activity $\mathrm{NADH}$ dehydrogenase activity oxidoreduclase activily, acting on $\mathrm{NAD}(\mathrm{P}) \mathrm{H}$

4 iron, 4 sulfur cluster binding aryl sulfotransferase activity calmodulin binding amino acid binding oxidorcductasc activity fructose 1,6-bisphosphate 1-phosphatase activity flavonol 3-sulfotransferase activity sulfotransferase activity

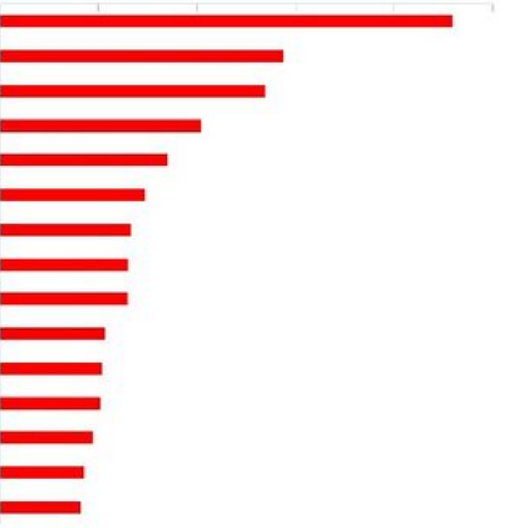


Figure 1

Significant Gene Ontology analysis of differentially expressed proteins. (BP, biological process; CC, Cellular component; MF, molecular function).
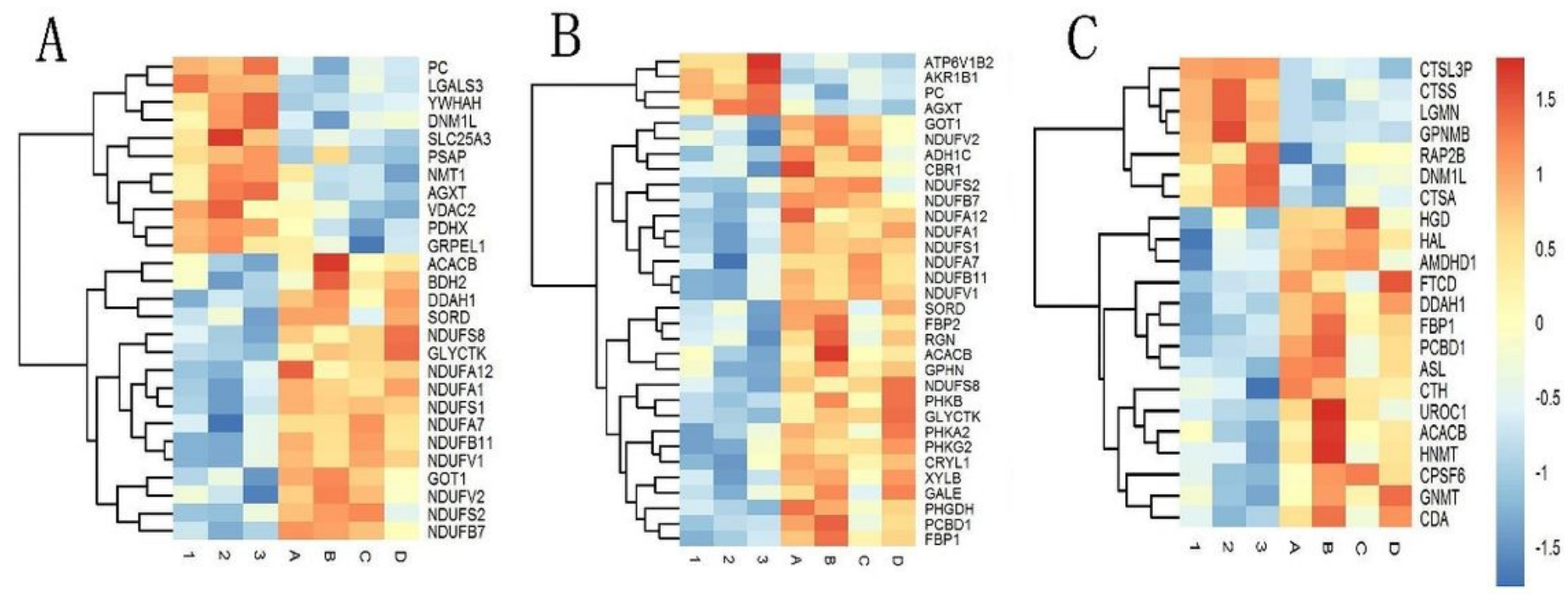

Figure 2

Heat map of differentially expressed proteins (DEPs) in PNALD and control patient. A: Mitochondrial and mitochondrial-related proteins; B: Glycolipid metabolisms associated proteins; C: Amino acid metabolisms associated proteins. Colored boxes represent up-regulation (red) and down-regulation (blue) in the PNALD. The color scale shown at the upper right indicates the fold changes in protein expression of all the samples. 1, 2, 3: PNALD group; A, B, C, D: Control group. 


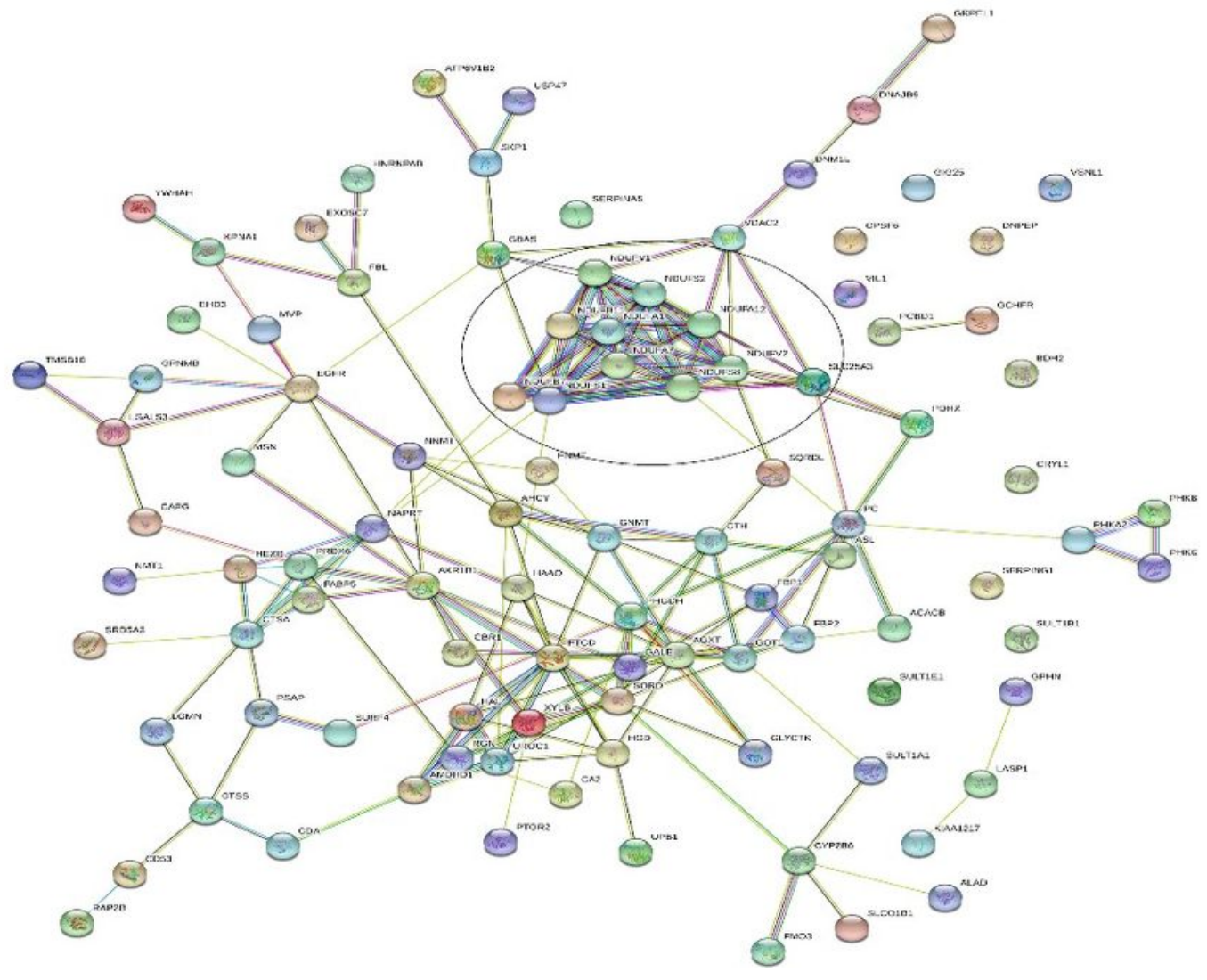

Figure 3

Protein-protein interaction of differentially-expressed protein for group PNALD vs. control

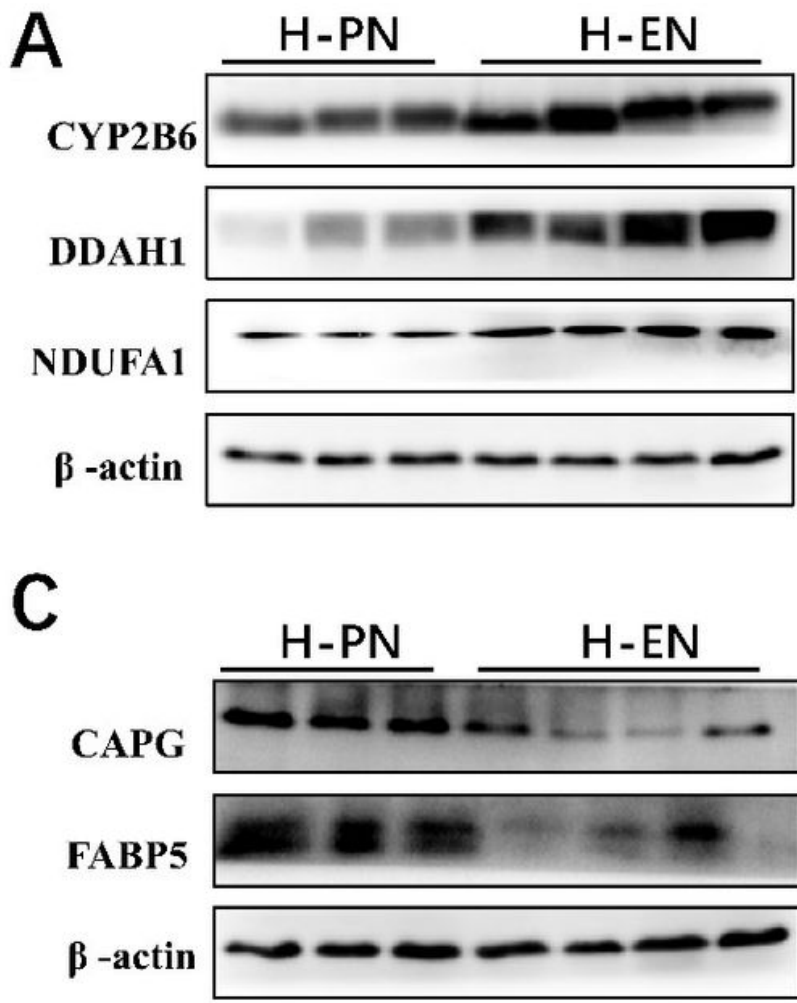

B
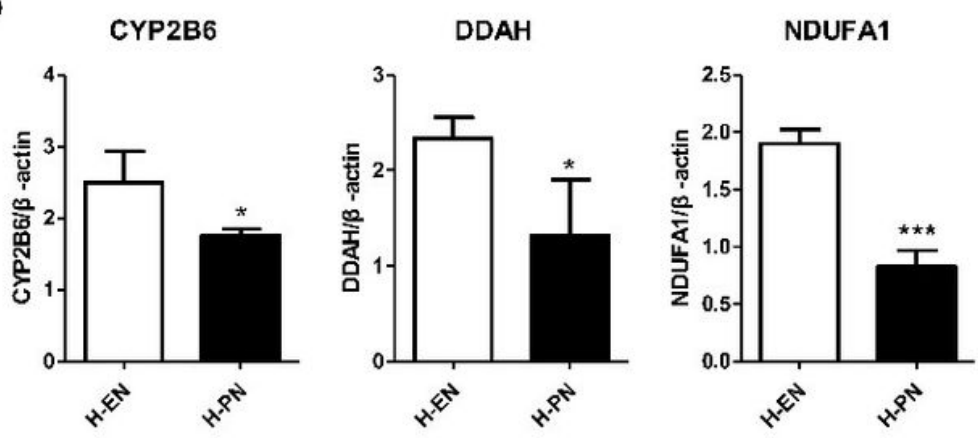

D

CAPG

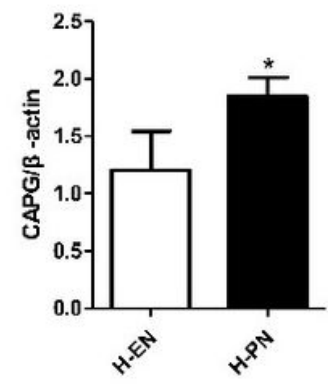

FABP5

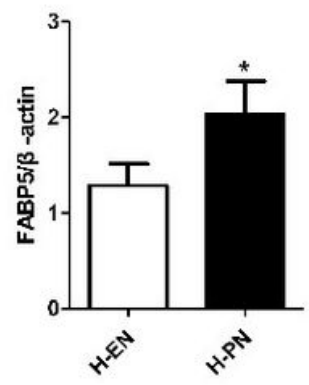


Figure 4

Confirmation of differentially expressed proteins (DEPs) by western blotting; A, C: Immunoblotting analysis of DEPs (CYP2B6, DDAH1, NDUFA1, CAPG, and FABP5) in PNALD(H-PN) and control (H-EN). B, D: Gray-Level scores. The $\beta$-actin protein was used as a control. *, Compared with the control group, $\mathrm{P}<$ $0.05 ; * \star \star$, Compared with the control group, $\mathrm{P}<0.001$.

\section{Supplementary Files}

This is a list of supplementary files associated with this preprint. Click to download.

- HierarchicalClusteringDataMatrix.xIsx

- Supplementaltables.docx

- Annotationenrichmentanalysis.xlsx

- DifferentProteins.xlsx

- NetworkAnalysisDegree.xlsx

- KEGGPathway.xIsx 